with an abnormal EEG. In 13 with abnormal and 17 with normal EEGs, the beneficial response rates were $61 \%$ and $88 \%$, respectively.

(Millichap JG. Recurrent headaches in 100 children.

Electroencephalographic abnormalities and response to phenytoin

(Dilantin). Child's Brain 1978;4:95-104).

\title{
MIGRAINE ASSOCIATED SYMPTOM PREVALENCE
}

A review of associated symptoms of migraine determined by telephone survey of 500 self-reported migraine sufferers is reported from Temple University School of Medicine and the Comprehensive Headache Center, Germantown Hospital, Philadelphia, PA. Female to male preponderance was 443 to $57 ; 5.6 \%$ were younger than 25 years old. Seventy-one percent took abortive medication, and $26,5 \%$ received both abortive and prophylactic medication. Precipitating factors included stress (79\%), changes in weather (44\%), premenstruation (37\%), changes in light (34\%), and eating certain foods $(30 \%)$. Symptoms associated with migraine attacks were headache (96\%), nausea/vomiting (32\%), photophobia (83\%), noise sensitivity (60\%), dizziness

- $(65 \%)$, eye pain, and neck pain (79\%). Nausea occurred in one half of attacks in $>90 \%$ of respondents, and vomiting in one third of attacks in $70 \%$, interfering with oral medication in $30 \%$ and $42 \%$, respectively. Many childhood migraineurs have a history of cyclical vomiting, a recognized precursor of migraine. (Silberstein SD. Migraine symptoms: Results of a survey of self-reported migraineurs. Headache July/August 1995;35:387-396). (Respond: Dr Stephen D Silberstein, The Comprehensive Headache Center, Germantown Hospital, One Penn Blvd, Philadelphia, PA 19144).

COMMENT. The authors emphasize the significance of nausea and vomiting as associated symptoms affecting the degree of disability of migraine sufferers. Drugs used to treat migraine often cause nausea and may exacerbate associated symptoms while relieving headache. The treatment or avoidance of nausea may be as important as the relief of headache. The route of administration of migraine medication can alter the prevalence of side-effects. Nausea and/or vomiting occur more frequently with oral administration of ergotamine or sumatriptan than with injection therapy.

Revisions to the International Headache Society

classification proposed for pediatric migraine proved more sensitive than existing criteria in a study of 45 children and adolescents at the Palm Beach Headache Center, Florida. For pediatric migraine without aura, the revisions included headache attacks as short as 30 minutes and a bilateral location in addition to unilateral headache. For those pediatric migraines with aura, the only proposed revision was a decrease in duration of headache from 2 - 48 hours to one half - 48 hours. Diagnostic rates increased from $53 \%$ to $80 \%$, using the revised criteria. (Winner P et al. Classification of pediatric migraine: Proposed revisions to the IHS criteria. Headache July/August 1995;35:407-410).

\section{HEADACHE AND STOMACH-ACHE CO-OCCURRENCE SYNDROME}

Psychosocial risk factors for headache and stomache-ache and their cooccurrence were investigated in a longitudinal study of Norwegian children aged 4-10 years at the Department of Health and Society, National Institute of Public Health, Oslo, Norway. Children with headache only were well behaved 
as preschoolers, high achievers, and their mothers were employed outside the home. The stomache-ache alone group had an earlier onset of symptoms than those with headache, they were well-adapted emotionally, and their mothers were less educated. Childhood emotional problems and low maternal emotional support differentiated the co-occurrence group from the headache and stomach-ache only groups. School factors were not associated with the cooccurrence syndrome. (Borge AIH, Nordhagen R. Development of stomachache and headache during middle childhood: co-occurrence and psychosocial risk factors. Acta Paediatr July 1995;84:795-802). (Respond: Dr AIH Borge, National Institute of Public Health, Geitmyrsveien 75, 0462 Oslo, Norway).

COMMENT. The co-occurrence of headache and stomach-ache in young children appears to constitute a distinct syndrome with psychosocial implications that may need to be addressed early to prevent later childhood incapacity. In the absence of central nervous or abdominal pathology, an interactive pediatric and psychological approach involving the mother and child is advised.

The absence of increased psychological or psychosocial disability in children with recurrent abdominal pain or in their families compared to controls, noted in the above study, is also alluded to in a letter to the editor (Feldman W. Recurrent abdominal pain in childhood. Acta Paediatr July 1995;84:834).

A study of esophogeal, gastric and duodenal biopsies in 31 children with migraine found twenty nine with an underlying inflammatory lesion of the gastrointestinal tract that could explain the associated symptoms of nausea (93\% of patients), vomiting (42\%), and abdominal pain (55\%). The authors suggest that the findings support a causal link between recurrent abdominal pain and migraine.

(Mavromichalis I et al. Migraine of gastrointestinal origin. Eur I Pediatr May 1995;154:406-410).

\section{SEIZURE DISORDERS}

\section{AUTONOMIC EPILEPSY REVIEWED}

In addition to gastointestinal manifestations of epilepsy, a review of autonomic epilepsies from the Deaconess and Beth Israel Hospitals and Harvard Medical School, Boston, MA, included cardiovascular manifestations, .sudden cardiac death, neurogenic pulmonary edema and other respiratory manifestations, diencephalic epilepsy, cutaneous manifestations, and urogenital manifestations. Gastrointestinal symptoms of epilepsy present as auras in adult patients with complex partial seizures, but ictal autonomic symptoms may be limited to visceral sensations, called abdominal epilepsy, especially in children. EEG abnormalities associated with ictal vomiting usually lateralize to the right or nondominant temporal lobe. (Freeman R, Schachter SC. Autonomic epilepsy. Seminars in Neurology June 1995;15:158166). (Reprints: Dr Freeman, Division of Neurology, Deaconess Hospital, Suite 7H, 110 Francis Street, Boston, MA 02215).

COMMENT. "Ictus emeticus and the nondominant temporal lobe" is discussed in Ped Neur Briefs June 1995;9:55. Some anticonvulsants have autonomic side-effects. (Millichap JG, Ortiz WR. Nitrazepam in myoclonic epilepsies. Am I Dis Child 1966;112:242-248). 